\title{
Composition and morphology of the follicular basal lamina during atresia of bovine antral follicles
}

\author{
H. F. Irving-Rodgers ${ }^{1 *}$, M. L. Mussard ${ }^{2 \dagger}$, J. E. Kinder ${ }^{2 \dagger}$ \\ and R. J. Rodgers ${ }^{1 *, \neq}$ \\ ${ }^{1}$ Department of Medicine, Flinders University of South Australia, Bedford Park, SA 5042, \\ Australia; and ${ }^{2}$ Department of Animal Sciences, University of Nebraska, Lincoln,
}

Nebraska 68583, USA

The fate of the follicular basal lamina during atresia was investigated using bovine follicles, in which different follicle phenotypes have been observed. These phenotypes include: healthy follicles with rounded basal granulosa cells with an aligned basal lamina or follicles with columnar basal granulosa cells with a basal lamina of many loops (loopy), and atretic follicles in which either the antral granulosa cells (antral atresia) or the basal cells (basal atresia) die first. Loopy lamina and basal atresia occur only in small antral follicles $<5 \mathbf{m m}$ in diameter. Follicles were collected from cattle of unknown reproductive history and processed for immunohistochemistry and electron microscopy, and from animals in which follicle growth had been monitored by daily measurements of follicle diameter by ultrasonography. Electron microscopic observations of dominant follicles during the growth phase, plateau and regression showed that the basal lamina was still visible and intact upon atresia. These follicles had a conventional aligned basal lamina, which they retained, except for some degree of folding, as they progressed into antral atresia. In small follicles (2-5 $\mathrm{mm}$ in diameter), the basal cell shape (rounded or columnar) and appearance of the basal lamina (aligned or of many loops) did not appear to be related to the type of atresia. On atresia the follicular basal laminae retained immunoreactive laminin $\alpha 1$ and $\beta 2$, type IV collagen $\alpha 1$ and nidogen. Laminin $\alpha 2$, which may come from the theca, was present in the follicular basal lamina of only $22 \%$ of healthy follicles, but was expressed very commonly in $71 \%$ of the atretic follicles. Laminin $\alpha 2$ expression was found in both phenotypes of healthy follicles, antral and basal atretic follicles, and follicles with aligned or loopy basal laminae. It is concluded that the basal lamina is not degraded upon atresia, but does undergo a variety of other changes.

\section{Introduction}

The mammalian ovary contains a pool of inactive primordial follicles. Each follicle is composed of a small inactive oocyte and a single layer of non-replicating granulosa cells enveloped by a basal lamina. In adults, a few primordial follicles become activated each day, the oocyte begins to enlarge and the granulosa cells divide. Layers of granulosa cells form and eventually a fluid-filled antrum develops in the centre of the follicle. Only large healthy antral follicles ovulate. Most of the follicles never reach this size and instead become atretic and regress.

Studies in many mammalian species have led to the concepts of follicle 'recruitment' and follicle 'dominance'. In bovine ovaries, follicle recruitment refers to a group of

*Current address: Reproductive Medicine Unit, Department of Obstetrics and Gynaecology, Adelaide University, Adelaide SA 5000, Australia

${ }^{+}$Current address: Department of Animal Sciences, The Ohio State University, Columbus, OH 43210-1095, USA

${ }^{\ddagger}$ Correspondence

Email: Ray.Rodgers@Adelaide.edu.au follicles growing at the same time and at a similar rate, as if in a wave. Follicle dominance refers to one follicle growing at a faster rate than other 'subordinate' follicles. These subordinate follicles eventually become atretic. If the dominant follicle is eliminated after atresia of the subordinate follicles has commenced, another cohort of growing follicles is recruited from the pool of smaller follicles. Two or three waves of follicles emerge in each oestrous cycle of cows. Follicles in each wave grow to about $5 \mathrm{~mm}$ in diameter before one dominant follicle emerges and enlarges to $>10$ $\mathrm{mm}$ in diameter. Follicles of this diameter are sufficiently large to ovulate if simulated by a surge release of $\mathrm{LH}$.

Non-ovulating follicles, including dominant follicles, undergo atresia. In our studies of atretic follicles, we have found that there are two forms of atresia: one in which cell death occurs initially in the basal region and spreads towards the antrum (basal atresia) and another in which the converse occurs (antral atresia) (Irving-Rodgers et al., 2001). Basal atresia occurs in antral follicles $<5 \mathrm{~mm}$ in diameter only. The structure of the membrana granulosa also varies among healthy follicles of this size (van Wezel et al., 1999).

In each follicle, the epithelial membrana granulosa is 
enveloped by a follicular basal lamina, which separates it from the surrounding stromal elements in primordial and preantral follicles, or from the specialized theca in antral follicles (van Wezel and Rodgers, 1996; Irving-Rodgers and Rodgers, 2000). There are two ultrastructural phenotypes of basal lamina in healthy bovine antral follicles $<5 \mathrm{~mm}$ in diameter. One phenotype is a conventional single layer, aligned closely to the base of the basal granulosa cells (Irving-Rodgers and Rodgers, 2000). The other phenotype is composed of many additional loops of basal lamina with associated matrix vesicles (Irving-Rodgers and Rodgers, 2000). Larger follicles (> $5 \mathrm{~mm}$ in diameter) have the former type only. The 'loopy' basal lamina occurs in healthy follicles with columnar basal cells and, conversely, the conventional aligned basal lamina occurs in follicles with rounded basal cells (Irving-Rodgers and Rodgers, 2000).

Basal laminae are often composed of type IV collagen, laminin and other matrix components. Importantly, basal laminae vary in composition from one epithelium to another, and each basal lamina will change in composition as the cells aligning it alter their behaviour. In follicles, the follicular basal lamina doubles 19-fold in surface area during follicular development (van Wezel and Rodgers, 1996) and changes in composition as it does so (Rodgers et al., 2000). During follicle growth, expression of type IV collagen $\alpha 3$ to $\alpha 6$ decreases, whereas $\alpha 1$ and $\alpha 2$ expression continues (Rodgers et al., 1998). Laminin $\beta 1$ is expressed transiently at the preantral stage and expression of laminin $\alpha 1, \beta 2$ and $\gamma 1$ increases (van Wezel et al., 1998). Nidogen and perlecan are not present in primordial follicles but are expressed later in follicular development (McArthur et al., 2000). Laminin $\alpha 2$ is very unusual; it is expressed in only a few healthy antral follicles and commonly in atretic follicles (van Wezel et al., 1998).

Conflicting observations and comments have been made about the effects of atresia on the follicular basal lamina. These range from suggestions that it is absent (Grimes et al., 1987) to enlarged to $>50 \mu \mathrm{m}$ (Singh and Adams, 2000). When the ultrastructure of the basal lamina has been observed during atresia it has not been the major focus of any study. Studies on the composition of the bovine follicular basal lamina have shown that some laminin chains $(\alpha 1, \alpha 2, \beta 2$ and $\gamma 1$ ) (van Wezel et al., 1998), the heparan sulphate proteoglycan, perlecan, and nidogen (McArthur et al., 2000) are present in atretic follicles. In the ovine follicular basal lamina of atretic follicles, Huet et al. (1997) also observed at least one of the components of laminin $1(\alpha 1, \beta 1$ or $\gamma 1)$, type IV collagen and heparan sulphate-containing proteoglycans. In the present study, an examination of the ultrastructure and composition of the follicular basal lamina during atresia was undertaken to advance our knowledge of the basal lamina and follicular atresia. Large dominant follicles were monitored by ultrasonography before harvesting and the smaller follicles, which cannot be monitored satisfactorily by this means in cattle, were collected from animals of unknown reproductive history.

\section{Materials and Methods}

\section{Tissues}

Bovine ovaries were collected from two different sources (see below) and handled and processed by a variety of methods (see below), thus increasing the probability of identifying possible artefacts produced by handling and processing. As the features observed were consistent between these different methods, the morphological features observed and described below were not artefacts of processing. The ovaries in groups 1 (small follicles) and 2 (large follicles) were harvested in such a way as to enable both immunohistochemical and electron microscopic examinations to be conducted on the same follicles, whereas ovaries in groups 3 and 4 were prepared for light and electron microscopy only.

Group 1. Bovine ovaries were collected at a local abattoir in South Australia, within 20 min of death, from mixed breeds of Bos taurus cows, assessed visually as not being pregnant. Ovaries were transported to the laboratory on ice $(n=87)$. Two follicles and adhering stroma $(2-5 \mathrm{~mm})$ were dissected from each ovary; their diameter was measured with the aid of an ocular micrometer in the eyepiece of a dissecting microscope and they were snap frozen in Tissue-Tek OCT embedding compound (Miles Inc., Elkhart, IN). The frozen follicles were bisected and one half was immersed in $2.5 \%(\mathrm{w} / \mathrm{v})$ glutaraldehyde, post-fixed in osmium tetroxide and embedded in epoxy resin (as described below). This portion was for subsequent light and electron microscopic assessment, and the remaining tissue in OCT compound was retained for subsequent immunohistochemical examination.

Group 2. Large follicles $(6-17 \mathrm{~mm}$ in diameter, one per ovary, $n=34$ ) were collected from the same source as group 1 and processed similarly. An additional 13 ovaries (two follicles were processed from two ovaries, and one follicle per ovary for the remainder) were snap frozen in OCT embedding compound. A total of 49 follicles was collected.

Group 3. Nine reproductive tracts were collected and one ovary per tract was perfusion fixed for electron microscopy, as described below. A total of 23 follicles $(2-5 \mathrm{~mm}$ in diameter) was examined in this manner. Follicle diameters were measured on tissue sections by averaging the maximum and minimum diameters from the position of the basal lamina.

Group 4. In the USA, cows aged 2.5 years, nulliparous and of composite breeding (one quarter Angus, one quarter Hereford, one quarter Pinzgauer and one quarter Red Poll) were used. Oestrus was synchronized by two i.m. injections of PGF $_{2 \alpha}$ (25 mg; Lutalyse; Pharmacia and Upjohn, Kalamazoo, MI) 10 days apart. During the first follicular wave of the next oestrous cycle (between day 7 and day 10 , 
day $0=$ oestrus) and once follicular dominance had been identified, all follicles $\geqslant 5 \mathrm{~mm}$ in diameter were aspirated using an Aloka 500V ultrasound machine attached to a $5 \mathrm{MHz}$ trans-vaginal aspiration probe. Aspiration initiated the synchronous emergence of a new wave of growing follicles, which was monitored by daily ultrasonography using a $7.5 \mathrm{MHz}$ trans-rectal probe attached to the Aloka $500 \mathrm{~V}$ machine. Ovaries were collected when the dominant follicle of the ensuing wave was in one of three development stages: (i) growing $(n=6)$ : had reached dominance, was $>8 \mathrm{~mm}$ in diameter and had not reached plateau stage; (ii) plateau $(n=4)$ : had reached dominance, was $>8 \mathrm{~mm}$ in diameter and had no growth for at least the $24 \mathrm{~h}$ before ovary collection, but was still functionally dominant as determined by the absence of new growth of follicular development; (iii) regression $(n=7)$ : had reached dominance, but was starting to regress in size, and emergence of a new wave of follicular development was evident. Ovariectomies were performed by laparotomy via para-lumbar incision and both ovaries were collected when possible.

\section{Processing of tissues for microscopy}

Portions of follicles from groups 1 and 2 (those fixed in glutaraldehyde only) were processed for electron microscopy. For groups 3 and 4, ovarian arteries were cannulated and the ovaries were flushed with $20 \mathrm{ml}$ medium (Earle's balanced salt solution for group 3 and Hank's solution for group 4), before flushing with $50 \mathrm{ml} 2.5 \%(\mathrm{w} / \mathrm{v})$ glutaraldehyde in buffer $(0.1 \mathrm{~mol}$ morpholinopropanesulphonic acid $\mathrm{I}^{-1}, \mathrm{pH} 7.3$, for group 3 or 0.1 mol phosphate buffer $\mathrm{I}^{-1}$, $\mathrm{pH} 7.3$, for group 4) delivered over 5-10 min. The ovaries were placed in fixative at $4^{\circ} \mathrm{C}$ overnight. Follicles were dissected from each ovary, cut in half and the crosssectional diameter was measured with the aid of an eyepiece ocular micrometer. For all groups, small pieces of the follicle wall were taken and placed in fixative, and stored at $4^{\circ} \mathrm{C}$. After several rinses with buffer to remove excess fixative, samples were post-fixed in $2 \%(\mathrm{v} / \mathrm{v})$ aqueous osmium tetroxide for $1 \mathrm{~h}$ at $4^{\circ} \mathrm{C}$, rinsed three times with distilled water ( 5 min each) and dehydrated by successive washes with acetone of increasing concentration to $100 \%$, on ice. After overnight infiltration with epoxy resin at room temperature, the specimens were embedded in fresh resin and polymerized at $60^{\circ} \mathrm{C}$ overnight. Sections $(1 \mu \mathrm{m}$ thickness) were stained with $1 \%(\mathrm{v} / \mathrm{v})$ aqueous methylene blue for light microscopic examination and $100 \mathrm{~nm}$ thick sections were stained with uranyl acetate and Reynold's lead citrate, and observed and photographed with a JEOL (Peabody, MA) CS1200 transmission electron microscope.

\section{Antibodies}

The primary antibodies for matrix molecules used were rabbit polyclonal antisera raised against human placental laminin $\alpha 2$ chain (M4; at 1:50 dilution; Lindblom et al., 1994), laminin 1 isolated from Englebreth Holm-Swarm (EHS) tumours (L9393; Sigma Chemical Co., St Louis, MO; at 1:100 dilution), purified nidogen isolated from mouse EHS tumour (913 or 914; 1:200 dilution; Dziadek et al., 1985) or recombinantly expressed mouse laminin $\alpha 1$ chain (317; Durbeej et al., 1996; at 1:100 dilution). Additional antibodies used were mouse monoclonal antibodies raised against bovine laminin $\beta 2$ chain (C4; at 1:100 dilution; Sanes and Chiu, 1983; Hunter et al., 1989), human laminin $\beta 1$ chain (clone 4E10; at 1:500 dilution) and laminin $\gamma 1$ chain (clone 2E8; at 1:500 dilution), both from Gibco BRL (Gaithersberg, MD). Rat monoclonal antibodies were raised against the non-collagenous 1 domain of each of the type IV collagen $\alpha 1$ to $\alpha 5$ chains (at 1:100 dilution; Sado et al., 1995) and $\alpha 6$ (at 1:100 dilution; Lees et al., 1998).

\section{Immunohistochemistry}

Portions of ovaries embedded in OCT compound were used for localization or co-localization using an indirect immunofluorescence method. Tissue sections $(10 \mu \mathrm{m}$ thickness) were cut from each of the frozen ovaries using a CM1800 Leica cryostat, and collected on glass slides treated with $0.01 \%(\mathrm{w} / \mathrm{v})$ poly L-ornithine hydrobromide (P-4638; Sigma Chemical Co.) and stored at $-20^{\circ} \mathrm{C}$ until use. Unfixed sections were dried under vacuum for $5 \mathrm{~min}$ followed by fixation in either $100 \%$ ethanol (in conjunction with anti-laminin $\alpha 2$ chain or anti-laminin 1) or $100 \%$ acetone (in conjunction with anti-laminin $\beta 2$ chain, antilaminin $\beta 1$ chain, anti-laminin $\gamma 1$ chain, anti-perlecan and anti-type IV collagen $\alpha 1$ to $\alpha 6$ chains), or unfixed (in conjunction with anti-nidogen). After fixation, sections were rinsed in three $\times 5$ min changes of hypertonic PBS (10 mmol sodium/potassium phosphate $\mathrm{I}^{-1}$ with $0.274 \mathrm{~mol}$ $\mathrm{NaCl} \mathrm{I-1}, 5$ mmol KCl I-1; $\mathrm{pH} 7.2 ; \mathrm{hPBS}$ ) before treatment with blocking solution (10\% normal donkey serum (D9663; Sigma Chemical Co.) in antibody diluent containing $0.55 \mathrm{~mol} \mathrm{NaCl} \mathrm{I}^{-1}$ and $10 \mathrm{mmol}$ sodium phosphate $\mathrm{I}^{-1}$, $\mathrm{pH}$ 7.1) for $20 \mathrm{~min}$ at room temperature. Incubation in primary antisera was carried out overnight at room temperature. The secondary antibodies used were fluorescein (DTAF)conjugated AffiniPure donkey anti-rabbit IgG (1:100; 711015-152) for the detection of nidogen, biotin-SP-conjugated AffiniPure donkey anti-mouse IgG (1:100; 715-066-151) for the detection of laminin $\gamma 1, \beta 1, \beta 2$ chains and perlecan, biotin-SP-conjugated AffiniPure donkey anti-rat $\lg \mathrm{G}$ (1:100; 712-066-153) for the detection of type IV collagen chains, and biotin-SP-conjugated AffiniPure donkey anti-rabbit $\lg G(1: 200 ; 711-066-152)$ for the detection of laminin $\alpha 1$ chains, and laminin 1 (EHS laminin), followed by Cy3conjugated streptavidin (1:100; 016-160-084), all from Jackson ImmunoResearch Laboratories Inc. (West Grove, $\mathrm{PA}$ ) in hypertonic PBS. Sections were mounted in mounting medium for fluorescence (S3023; Dako, Botany).

\section{Light microscopic observations and photography}

Sections of bovine ovary stained with methylene blue were examined using an Olympus BX50 microscope, with or without Nomarski optics, and photographed with an 
Table 1. Proportion of group 1 (2-5 mm in diameter) bovine follicles (per number examined) classified as healthy with rounded or columnar basal cells, or antral or basal atretic at early, mid- or late stages of atresia that contained matrix components

\begin{tabular}{|c|c|c|c|c|c|c|c|}
\hline \multirow[b]{2}{*}{ Matrix component } & \multicolumn{2}{|c|}{ Healthy } & \multicolumn{2}{|c|}{ Basal atresia } & \multicolumn{3}{|c|}{ Antral atresia } \\
\hline & Rounded & Columnar & Mid- & Advanced & Early & Mid- & Advanced \\
\hline \multicolumn{8}{|l|}{ Type IV collagen } \\
\hline$\alpha 1$ & $1 / 1$ & $4 / 4$ & $8 / 8$ & $4 / 4$ & $4 / 4$ & $2 / 2$ & $3 / 3$ \\
\hline$\alpha 3$ & $0 / 0$ & $0 / 3$ & $0 / 4$ & $0 / 2$ & $0 / 5$ & $0 / 3$ & $0 / 5$ \\
\hline$\alpha 4$ & $0 / 1$ & $0 / 4$ & $0 / 4$ & $0 / 4$ & $0 / 5$ & $0 / 3$ & $0 / 5$ \\
\hline$\alpha 5$ & $0 / 0$ & $0 / 3$ & $0 / 4$ & $0 / 4$ & $0 / 5$ & $0 / 4$ & $0 / 5$ \\
\hline$\alpha 6$ & - & - & $1 / 6$ & $0 / 3$ & $0 / 5$ & $0 / 3$ & $0 / 4$ \\
\hline \multicolumn{8}{|l|}{ Laminin } \\
\hline$\alpha 1$ & $0 / 0$ & $4 / 4$ & $0 / 2$ & - & - & $0 / 1$ & $1 / 1$ \\
\hline$\alpha 2$ & $1 / 11$ & $3 / 7$ & $6 / 10$ & $5 / 7$ & $6 / 6$ & 7/12 & $6 / 7$ \\
\hline$\beta 2$ & $1 / 1$ & $4 / 4$ & $8 / 8$ & $4 / 4$ & $5 / 5$ & $2 / 3$ & $5 / 5$ \\
\hline Nidogen & $11 / 11$ & $7 / 7$ & $4 / 4$ & $7 / 7$ & $8 / 8$ & $11 / 11$ & $8 / 8$ \\
\hline
\end{tabular}

Olympus SC35 camera attachment and Ilford FP-4 125 black and white film. Sections stained immunohistochemically were observed and photographed with an Olympus Vanox AHBT3 epifluorescence microscope with Olympus C35AD-4 camera attachment and photographed with Kodak T-Max 400 black and white film.

\section{Classification of follicles by light microscopy}

Follicles were assessed as healthy or atretic. Atretic follicles were assessed as undergoing basal or antral atresia (Irving-Rodgers et al., 2001), and divided further into early, mid- or late stages on the basis of the degree of cellular degeneration during atresia.

\section{Results}

\section{Classification of follicles}

Follicles were classified as either healthy or atretic on the basis of their morphology, using epoxy resin-embedded material where available or frozen sections. Healthy follicles were classified as having either columnar or rounded basal cells (Irving-Rodgers and Rodgers, 2000).

The atretic follicles were classified further as undergoing either antral or basal atresia (Irving-Rodgers et al., 2001). Atretic follicles were further classified arbitrarily as early, mid- or late atretic. Early antral atretic follicles had pyknotic nuclei in the antra, mid-atretic follicles had pyknotic nuclei in the antral layers and late atretic follicles had advanced pyknosis, such that only 1-2 layers of basal healthy cells remained. Basal atresia was classified as mid- or late; early stages were difficult to differentiate from healthy follicles using light microscopy. Cell death in mid-basal atretic follicles occurred in the basal layer with partial expansion of matrix there. In late basal atresia, extensive basal areas of the former membrana granulosa were occupied by fluid, cell debris and macrophages.

\section{Classification of follicles in groups 1 and 2}

In group 1, 139 of 174 follicles were examined: 91 follicles were healthy (65\%), 29 were undergoing antral atresia (21\%) and 19 follicles were undergoing basal atresia (14\%). Of these 139 follicles, 68 were taken for immunohistochemistry, and the mean \pm SEM sizes of the follicles were similar among the healthy (3.5 $\pm 0.2 \mathrm{~mm} ; n=20)$, basal atretic $(3.5 \pm 0.2 \mathrm{~mm} ; n=19)$ and antral atretic $(3.8 \pm 0.1 \mathrm{~mm} ; n=29)$ follicles. Classification of healthy follicles used for immunohistochemistry into columnar or rounded basal granulosa cells is shown (Table 1). Of the early, mid- and late antral atretic follicles, 3/6, 10/12 and $6 / 6$ had rounded basal cells, respectively. The other follicles had columnar cells, except one of the early follicles, which was intermediate in appearance. In group 2, 44 follicles were examined: 28 were healthy $(n=19$ follicles in 6-10 $\mathrm{mm}$ range, mean $8.6 \pm 0.4 \mathrm{~mm}$ in diameter; and $n=9$ follicles in $12-17 \mathrm{~mm}$ range, mean $14.7 \pm 1.7 \mathrm{~mm}$ in diameter); 16 were undergoing antral atresia (all 6-10 mm in diameter, mean $7.8 \pm 0.4 \mathrm{~mm}$ in diameter) and none were undergoing basal atresia.

\section{Composition of the basal lamina in groups 1 and 2}

Immunostaining for nidogen was used extensively for localizing the follicular basal lamina. The follicular basal lamina was present in all follicles examined in group 1, regardless of whether they were healthy with rounded or columnar basal cells (Table 1) or were undergoing antral or basal atresia (Table 1). Similarly, in group 2, nidogen was present in the follicular basal lamina of all the healthy (13/13 in $6-10 \mathrm{~mm}$ and $9 / 9$ in $12-17 \mathrm{~mm}$ follicles) and antral atretic (10/10 for $6-10 \mathrm{~mm}$ follicles) follicles examined by immunostaining for nidogen.

As shown by Rodgers et al. (1998), the follicular basal laminae of healthy antral follicles of the sizes examined in the present study contained type IV collagen $\alpha 1$, but not $\alpha 3$ 

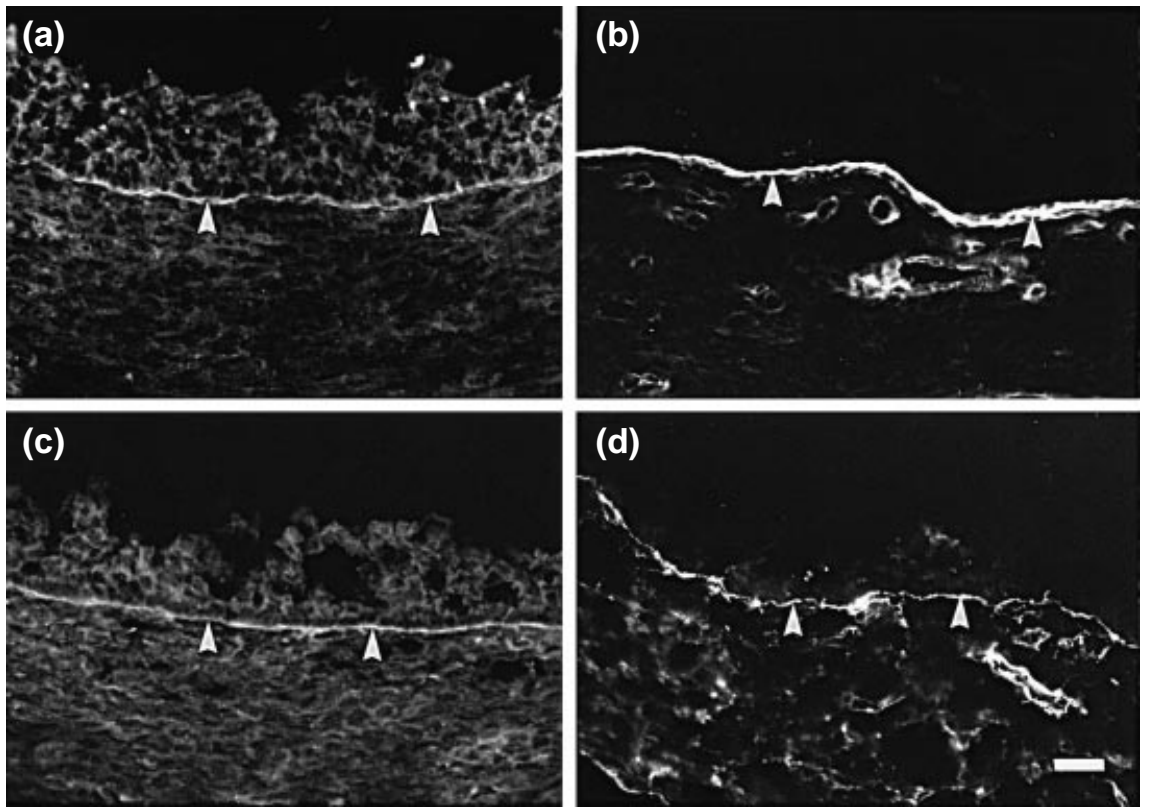

Fig. 1. Immunolocalization of laminin $\beta 2$ in (a) healthy (2 mm), (b) late basal ( $5 \mathrm{~mm})$ and (c) early antral $(4 \mathrm{~mm}$ ) atretic bovine follicles, and (d) type IV collagen $\alpha 1$ in a late basal atretic follicle $(4.5 \mathrm{~mm})$. Follicular antrum is towards the top of each panel. Arrowheads indicate basal lamina. Scale bar represents $20 \mu \mathrm{m}$.

to $\alpha 6$ (Table 1 ); $\alpha 2$ was not examined in the present study. The composition of collagens in the basal lamina did not change during either antral or basal atresia (Table 1 and Fig. 1).

Laminin $\alpha 1$ and $\beta 2$ (Fig. 1) were present in both healthy and atretic follicles (van Wezel et al., 1998) of the sizes examined in the present study. The composition did not change with respect to these laminin chains during either antral or basal atresia. In agreement with previous observations, where laminin $\alpha 2$ was expressed more highly in atretic follicles (van Wezel et al., 1998), laminin $\alpha 2$ was detected in 4/18 healthy follicles examined only (Table 1) compared with 27/41 atretic follicles. Laminin $\alpha 2$ was found in both basal and antral atretic follicles (Table 1 and Fig. 2b,d,e). As there was some diversity in the expression of laminin chains in both healthy and atretic follicles, the follicular basal laminae were examined by electron microscopy. The pattern of expression was independent of whether the follicular basal lamina was a conventional aligned basal lamina or a 'loopy' basal lamina (Table 2).

\section{Morphology of the basal lamina in groups 1, 2 and 3}

The follicular basal lamina was observed as either a single layer of electron-dense material or with additional loops of basal lamina as described by Irving-Rodgers and Rodgers (2000). Follicles undergoing antral atresia (Figs 3 and 4) had either an aligned (Figs 3 and 4b) or loopy basal lamina (Fig. 4a), irrespective of whether the tissue was fixed by perfusion (Figs $3 \mathrm{a}$ and 4b) or immersion (Fig. 3b).
Table 2. Numbers of healthy or antral or basal atretic bovine follicles in group 1 with (positive) or without (negative) laminin $\alpha 2$ in the follicular basal lamina which were either of 'loopy' or aligned phenotype

\begin{tabular}{|c|c|c|c|c|}
\hline Follicle type & & Laminin $\alpha 2$ & Loopy & Aligned \\
\hline \multirow[t]{2}{*}{ Healthy } & & Positive & 1 & 1 \\
\hline & & Negative & 3 & 1 \\
\hline \multirow[t]{2}{*}{ Basal atresia } & Mid- & Positive & 3 & 2 \\
\hline & Late & Positive & 2 & 3 \\
\hline \multirow[t]{4}{*}{ Antral atresia } & Mid- & Positive & 2 & 2 \\
\hline & & Negative & 3 & 3 \\
\hline & Late & Positive & 2 & 2 \\
\hline & & Negative & 3 & 0 \\
\hline
\end{tabular}

Similarly, basal atretic follicles had either an aligned (Fig. 5a) or loopy basal lamina (Fig. 5b). The basal lamina often appeared convoluted late in antral atresia (Fig. 4b). Regressing follicles had an extensively folded basal lamina, which was often traversed by macrophages, endothelial cells and fibroblasts (Fig. 6).

\section{Growth parameters and classification of group 4 follicles}

Full details of the growth parameters of these follicles have been reported by Irving-Rodgers et al. (2001). In brief, follicles in each of the three groups were harvested at different intervals during the follicle wave. Follicles were harvested at $3.7 \pm 0.7,7.5 \pm 1.7$ and $8.3 \pm 0.9$ days after 

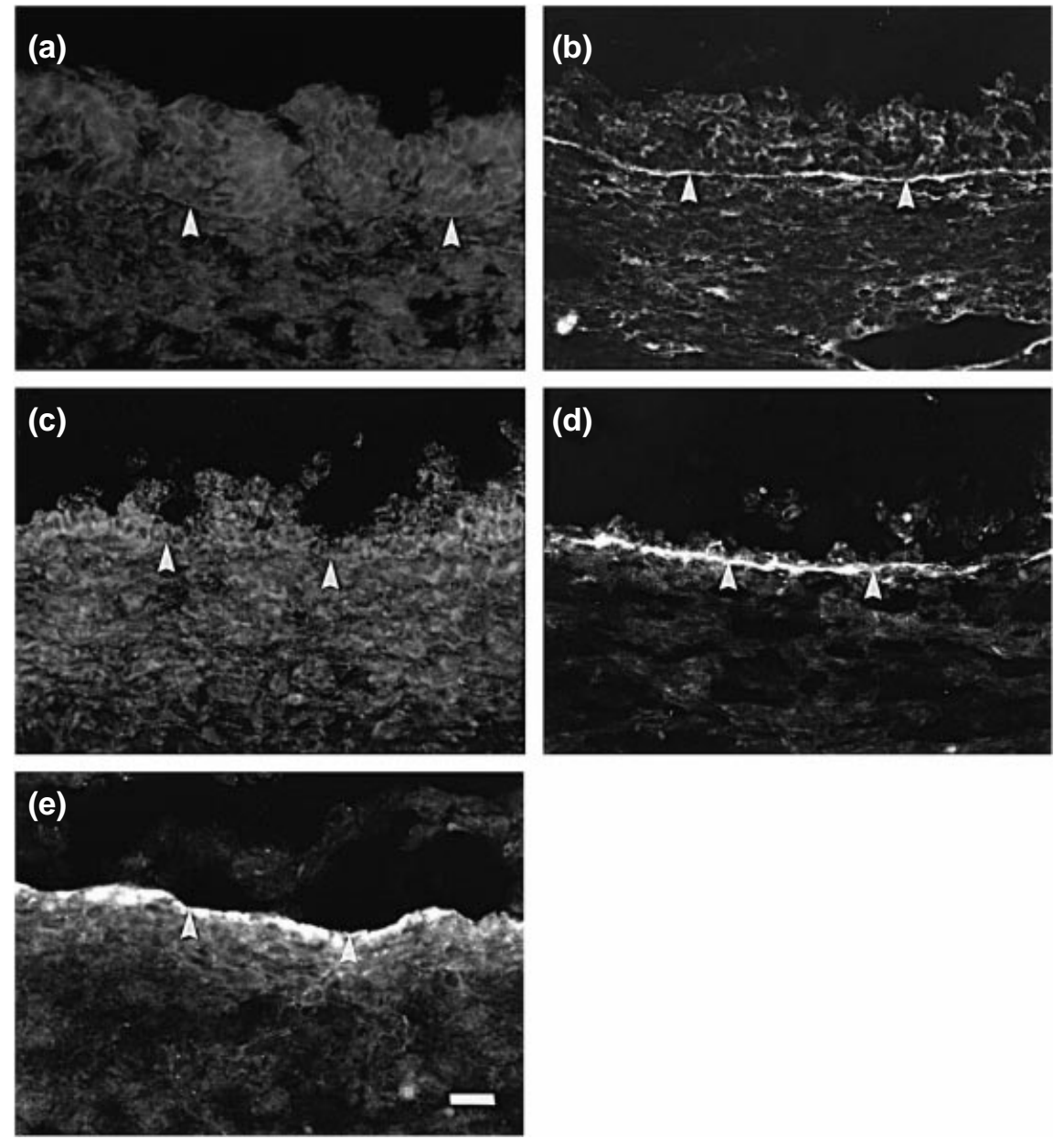

Fig. 2. Immunolocalization of laminin $\alpha 2$ in (a) healthy (2 $\mathrm{mm})$ or (c) antral atretic $(4.5 \mathrm{~mm})$ bovine follicles, which do not have detectable staining, and (b) early antral $(4 \mathrm{~mm}),(\mathrm{d})$ late antral $(3.5 \mathrm{~mm})$ or (e) late basal $(3 \mathrm{~mm})$ atretic follicles, which do stain positively. Follicular antrum is towards the top of each panel. Arrowheads indicate basal lamina. Scale bar represents $20 \mu \mathrm{m}$.

reaching a size of $5 \mathrm{~mm}$ for the growing, plateau and regressing groups, respectively. Maximal growth rates attained before harvesting (growing subgroup) or on reaching dominance (plateau and regressing subgroups) were similar for the three subgroups $(1.2 \pm 0.4,1.0 \pm 0.6$ and $1.5 \pm 0.5 \mathrm{~mm}$ day $^{-1}$, respectively), indicating that the three subgroups of follicles followed similar growth trajectories as they assumed dominance. The sizes of the follicles at harvesting were similar between the subgroups $(9.1 \pm 0.6$, $9.9 \pm 0.5$ and $9.0 \pm 0.9 \mathrm{~mm}$ for growing, plateau and regressing follicles, respectively), but clearly the growth just before collection was significantly different $(P<0.05$; ANOVA and SNK tests; $1.2 \pm 0.4,0.0 \pm 0.0$ and $-0.6 \pm 0.1 \mathrm{~mm} \mathrm{day}^{-1}$, respectively). Thus, the follicles in each of the three groups were similar except they were at different stages of dominance or regression.

Of the growing dominant follicles, five follicles had a healthy granulosa layer and no or very few pyknotic nuclei; basal granulosa cells were rounded in shape. One follicle had cells with pyknotic nuclei located near the antrum. All of the plateau dominant follicles had rounded granulosa cells; pyknotic nuclei were observed on the antral aspect of the membrana granulosa (2/4 follicles) or among basal cells (one follicle). The regressing dominant follicles were atretic, five follicles had rounded granulosa cells; the membrana granulosa of the other two follicles consisted of flattened cells. All atretic follicles fit the description of antral atretic follicles except that the granulosa cells of dominant atretic follicles were flattened in comparison to small follicles and even follicles $10 \mathrm{~mm}$ in diameter.

\section{Ultrastructure of the follicular basal lamina in group 4}

All follicles examined, irrespective of their stage of growth or atresia, had a basal lamina that was a single layer and aligned closely to the membrana granulosa. As atresia progressed, granulosa cells became detached from the basal 

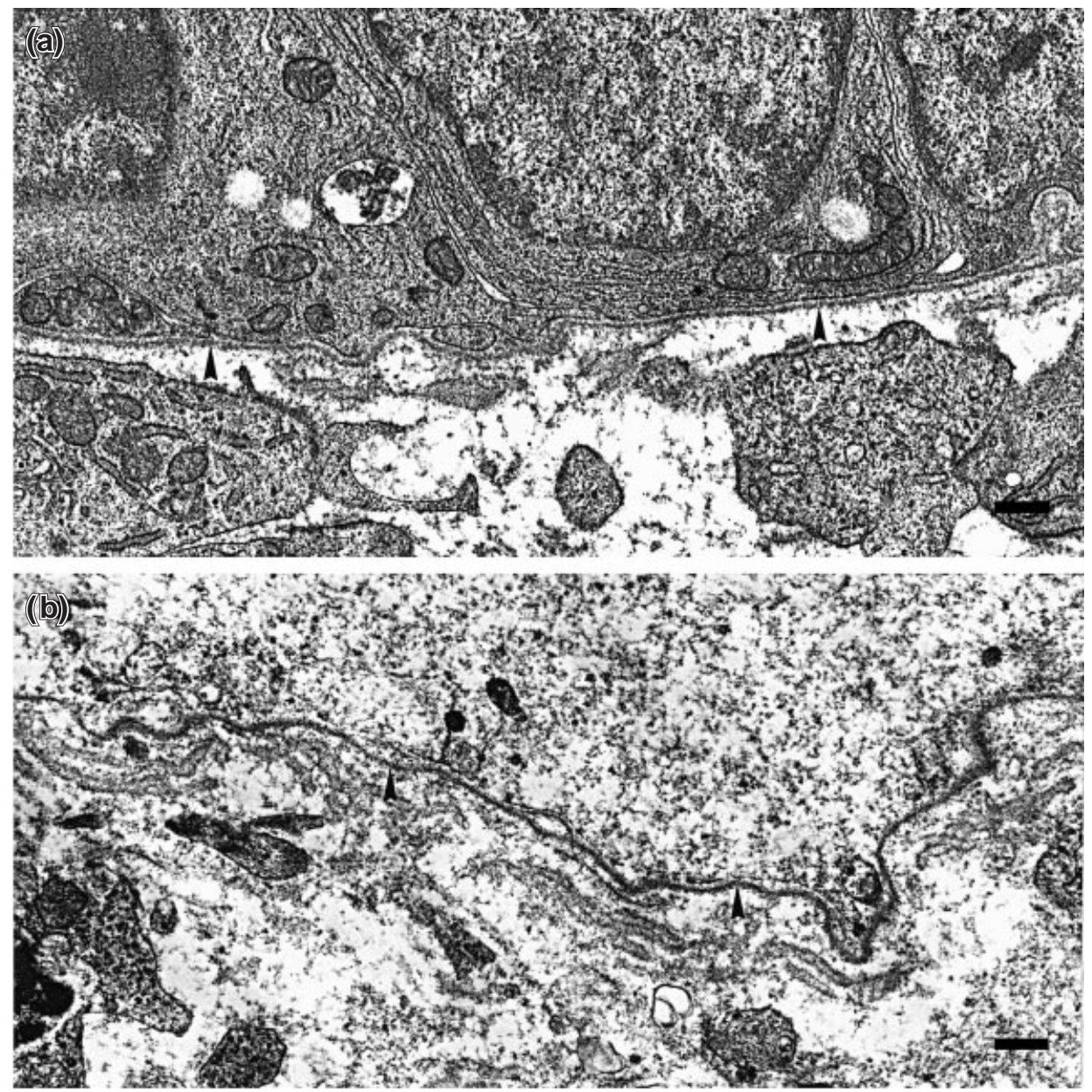

Fig. 3. Electron micrograph of basal lamina from antral atretic bovine follicles. (a) Early antral atresia, perfusion fixed, aligned basal lamina, follicle $6.5 \mathrm{~mm}$ in diameter. (b) Late antral atresia, immersion fixed, aligned basal lamina, follicle $4 \mathrm{~mm}$ in diameter. Follicular antrum is towards the top of each panel. Arrowheads indicate basal lamina. Scale bar represents $200 \mathrm{~nm}$.

lamina (Fig. 4b), which was 'concertina-like' in appearance and exposed to the follicular fluid. However, the basal lamina remained intact and there was no evidence of degradation as either thinning of the basal lamina or breaches in the basal lamina.

\section{Discussion}

The results of the present study show that on atresia of follicles, regardless of the type of atresia, the basal lamina is not degraded. Electron microscopic observations found that the basal lamina is still visible and intact, and retains immunoreactive laminin $\alpha 1$ and $\beta 2$, and type IV collagen $\alpha 1$ and nidogen. Laminin $\alpha 2$ is expressed more commonly in atretic follicles than in healthy follicles, but its expression does not appear to be related to either type of atresia or the degree of atresia. Although large healthy follicles have a conventional aligned basal lamina which they retain as they progress into antral atresia, the smaller follicles on atresia have either the aligned or loopy type, irrespective of whether the atresia process first involves death of antral or basal cells.

In general, basal laminae can undergo many changes, not just in composition as occurs with follicle development (Rodgers et al., 1998; van Wezel et al., 1998; McArthur et al., 2000). For example, diabetics often have one thickened basal lamina or one of many layers (Vracko, 1974; Martinez-Hernandez and Amenta, 1983; Abrahamson, 1986). Basal laminae are often degraded on epithelialmesenchymal transition, as occurs to granulosa cells on ovulation. The event of atresia as it occurs in follicles is unusual, as it involves complete destruction of the epithelial layer by death of all the epithelial cells. In other epithelia, such as luminal gut epithelium or involuting glands, cells undergo apoptosis as they age, but this is regional and involves only a few cells at any one time. Certainly the stem 


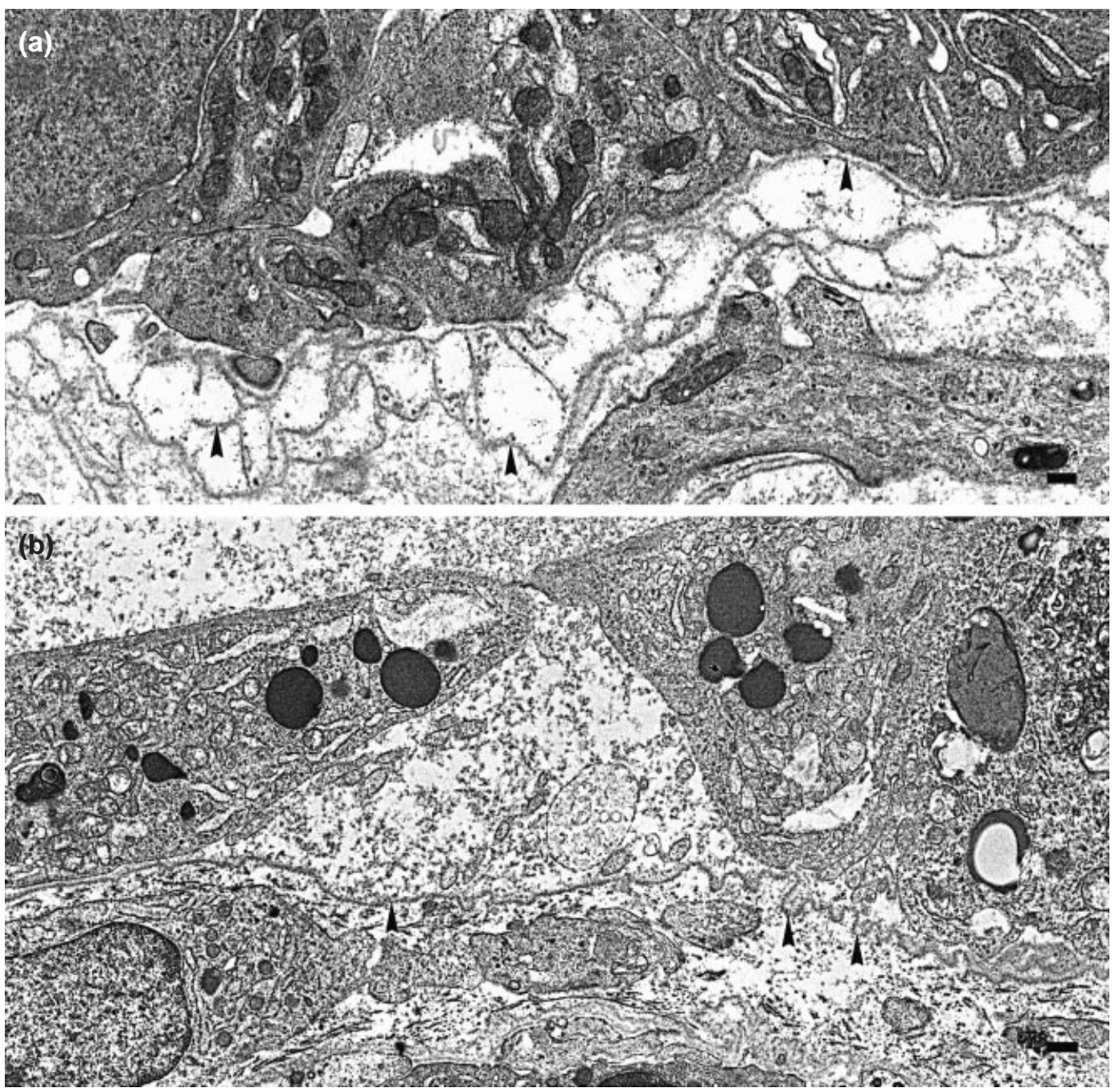

Fig. 4. Electron micrograph of basal lamina from antral atretic bovine follicles. (a) Early antral atresia, immersion fixed, loopy basal lamina, follicle $3 \mathrm{~mm}$ in diameter. (b) Antral regressing follicle, perfusion fixed, aligned basal lamina, $7 \mathrm{~mm}$ in diameter. Follicular antrum is towards the top of each panel. Arrowheads indicate basal lamina. Scale bar represents $500 \mathrm{~nm}$.

cells survive to regenerate the populations of cells. However, the stem cells of the membrana granulosa (Lavranos et al., 1999; Rodgers et al., 1999, 2001) do not survive follicle atresia. Thus, the study of follicular atresia and the changes in the follicular basal lamina pose unique questions.

Very little information has been published on the follicular basal lamina during atresia, but there have been passing comments. For example, Grimes et al. (1987) described atretic follicles as having '... a sparse or almost absent granulosa layer, a few squamous cells among the theca which varies in thickness and loosely arranged and disorganized and absent basement membrane...'. Singh and Adams (2000) used light microscopy to show that the basal lamina was enlarged to up to $50 \mu \mathrm{m}$. As this is 1000fold thicker than conventional basal laminae, they may in fact have been referring to the region visible between the theca and the basal layers of granulosa cells. From the present study it is clear that the basal lamina is not degraded or thickened. Electron microscope studies show that as the follicle shrinks, folding of the basal lamina does occur, but this is not thickening as can occur in diabetic basal laminae. This folding becomes more extensive as follicles continue to regress. Thus, complete destruction of the follicular basal lamina is not one of the early events of atresia.

Even if the basal lamina is not destroyed completely early in atresia, it is clear that cells from the theca can breach it (Bagavandoss et al., 1983). Macrophages, endothelial cells and fibroblasts have been observed breaching the follicular basal lamina as they migrate from the thecal layer of atretic follicles. In a more detailed study, Irving-Rodgers et al. (2001) found that macrophages were not present in the membrana granulosa of healthy follicles or in antral atretic 

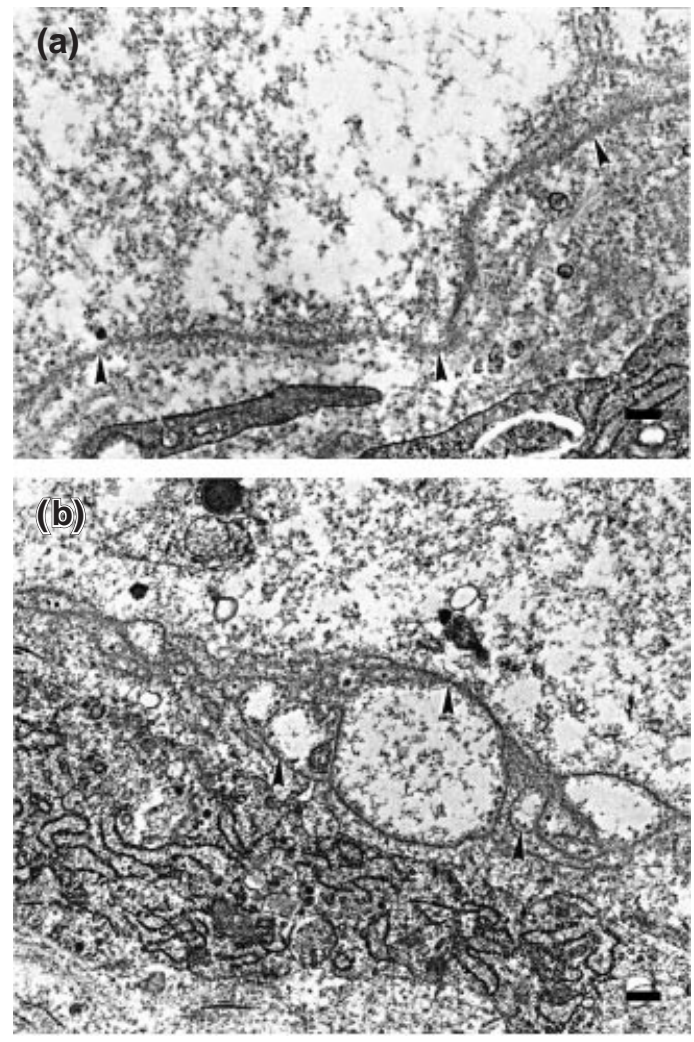

Fig. 5. Electron micrograph of basal lamina from basal atretic bovine follicles. (a) Late basal atresia, immersion fixed, aligned basal lamina, follicle $5 \mathrm{~mm}$ in diameter. (b) Mid-basal atresia, immersion fixed, loopy basal lamina, follicle $3.5 \mathrm{~mm}$ in diameter. Follicular antrum is towards the top of each panel. Arrowheads indicate basal lamina. Scale bars represent (a) 250 and (b) $200 \mathrm{~nm}$.

follicles until late in atresia, when most of the cells aligning the basal lamina had died. In basal atresia, where cells aligning the basal lamina die first, macrophages often breach the follicular basal lamina (Irving-Rodgers et al., 2001). Thus, movement of cells from the theca is restrained while the follicular basal lamina is aligned with healthy granulosa cells. However, wholesale degradation of the basal lamina does not occur.

As reported in the present study and by van Wezel et al. (1998), laminin $\alpha 2$ is expressed in some healthy follicles and in a high proportion of, but not all, atretic follicles. In the present study it was found that laminin $\alpha 2$ expression was not related to the different phenotypes of basal granulosa cells (rounded or columnar), basal lamina (aligned or loopy) or atresia (basal or antral). Of the laminin $\alpha$ isoforms, $\alpha 2$ is often associated with non-epithelial basal laminae, such as are found in smooth, cardiac or skeletal muscles, or in capillaries or nerves (Jucker et al., 1996; Oliviero et al., 2000; Vajsar et al., 2000). Where laminin $\alpha 2$ is associated with the basal lamina of epithelia it may be of mesenchymal origin (Sorokin et al., 1997; Lefebvre et al., 1999). This may be the case in follicles too. Why it is expressed in only some healthy antral follicles and a high

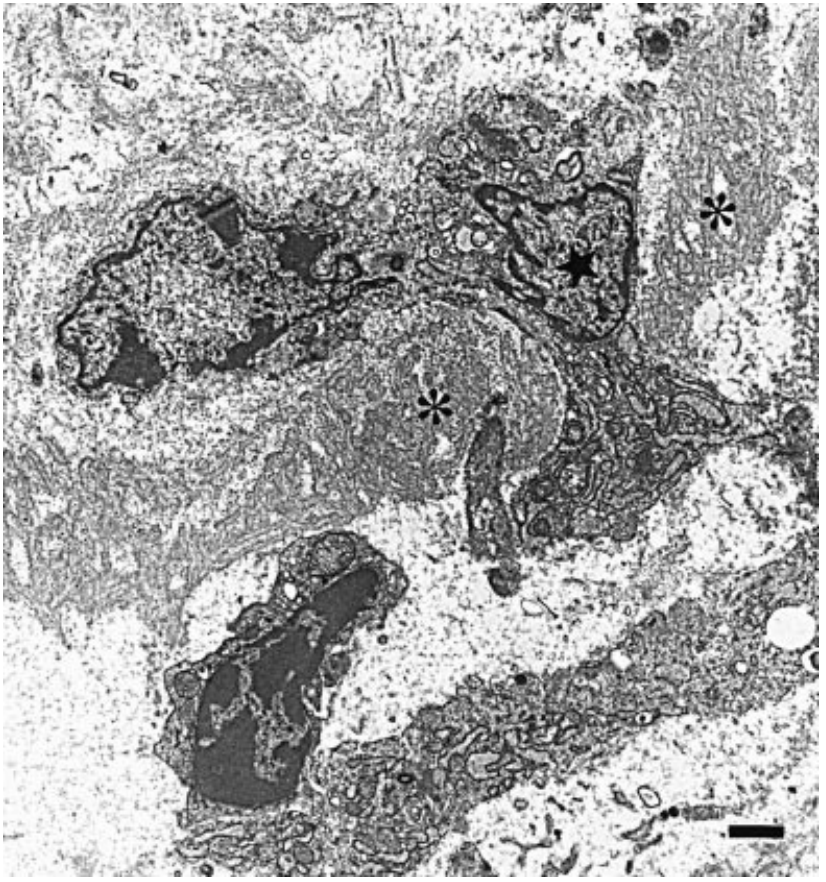

Fig. 6. Electron micrograph of a late basal atretic bovine follicle showing highly convoluted basal lamina, being breached by a fibroblast. Follicular antrum is towards the top. Immersion fixed follicle $2 \mathrm{~mm}$ in diameter. Asterisks indicate basal lamina. Star indicates a fibroblast. Scale bar represents $1 \mu \mathrm{m}$.

proportion, but not all, atretic follicles has yet to be determined.

In small healthy follicles ( $<5 \mathrm{~mm}$ in diameter), two types of basal lamina have been found, aligned or loopy, and these occur in follicles which have rounded or columnar basal granulosa cells, respectively (Irving-Rodgers and Rodgers, 2000). In larger antral follicles (>5 mm in diameter), only an aligned basal lamina with rounded basal cells has been observed (Irving-Rodgers and Rodgers, 2000). In agreement in the present study, the large dominant follicles had rounded cells and an aligned basal lamina. On atresia they underwent antral atresia and retained an aligned basal lamina. In smaller follicles there was no link between basal lamina phenotype and atresia type, as there was in large follicles. Thus, one of two events had occurred in the small follicles. Both types of small healthy follicles could have undergone either type of atresia, unlike large follicles. Alternatively, if healthy follicles with a rounded basal cell phenotype underwent antral atresia and those with a columnar phenotype underwent basal atresia, the phenotype of the basal lamina must have changed on atresia.

In conclusion, the follicular basal lamina survives the early events of atresia, retaining the components that were present before atresia. Its ultrastructure and composition can change upon atresia. However, as these changes do not occur uniformly in every atretic follicle it is unlikely that the follicular basal lamina is a key initiator of events in early 
atresia. Instead, it appears that the events of atresia have an effect on the basal lamina, which does not involve degradation.

This work was supported by the Flinders Medical Centre Research Foundation, the Flinders University of South Australia and the National Health and Medical Research Council of Australia, the USDA CRGO 90-37240-5724 and appropriated funding of the State of Nebraska. The authors would like to thank Ingrid van Wezel for discussions on the morphology of the follicular basal lamina during atresia. The technical assistance of Deb Clopton, Ken Pearson and Candy Toombs in ovary collection in the USA is much appreciated. The authors would like to thank the following for kind donations of antibodies: Marie Dziadek of the Department of Anatomy, University of Melbourne for anti-nidogen (\#913, \#914); Mats Paulsson of the Institute for Biochemistry, Medical Faculty, University of Cologne, Germany for anti-laminin $\alpha 2$ (\#M4); Lydia Sorokin of the Institute for Experimental Medicine, University of Erlangen-Nurnburg, Germany for anti-laminin $\alpha 1$; and Yoshikazu Sado, Division of Immunology, Shigei Medical Research Institute in Okayama and Yoshifumi Ninomyia from the Department of Molecular Biology and Biochemistry, Okayama University Medical School, for anti type IV collagens.

\section{References}

Abrahamson D (1986) Recent studies on the structure and pathology of basement membranes Journal of Pathology 149 257-278

Bagavandoss P, Midgley AR and Wicha M (1983) Developmental changes in the ovarian follicular basal lamina detected by immunofluorescence and electron microscopy Journal of Histochemistry and Cytochemistry $31633-640$

Durbeej M, Fecker L, Hjalt T et al. (1996) Expression of laminin $\alpha 1, \alpha 5$ and $\beta 2$ chains during embryogenesis of the kidney and vasculature Matrix Biology 15 397-413

Dziadek M, Paulsson M and Timpl R (1985) Identification and interaction repertoire of large forms of the basement membrane protein nidogen EMBO Journal 4 2513-2518

Grimes RW, Matton P and Ireland JJ (1987) A comparison of histological and non-histological indices of atresia and follicular function Biology of Reproduction 37 82-88

Huet C, Monget P, Pisselet C and Monniaux D (1997) Changes in extracellular matrix components and steroidogenic enzymes during growth and atresia of antral ovarian follicles in the sheep Biology of Reproduction 56 1025-1034

Hunter DD, Shah V, Merlie JP and Sanes JR (1989) A laminin-like adhesive protein concentrated in the synaptic cleft of the neuromuscular junction Nature 338 229-234

Irving-Rodgers HF and Rodgers RJ (2000) Ultrastructure of the follicular basal lamina of bovine ovarian follicles and its relationship to the membrana granulosa Journal of Reproduction and Fertility 118 221-228

Irving-Rodgers HF, van Wezel IL, Mussard ML, Kinder JE and Rodgers RJ (2001) Atresia revisited: two basic patterns of atresia of bovine antral follicles Reproduction 122 761-775

Jucker M, Tian M, Norton DD, Sherman C and Kusiak JW (1996) Laminin alpha 2 is a component of brain capillary basement membrane: reduced expression in dystrophic dy mice Neuroscience 71 1153-1161

Lavranos TC, Mathis JM, Latham SE, Kalionis B, Shay JW and Rodgers RJ (1999) Evidence for ovarian granulosa stem cells: telomerase activity and localisation of the telomerase RNA component in bovine ovarian follicles Biology of Reproduction 61 358-366
Lees GE, Helman RG, Kashtan CE et al. (1998) A model of autosomal recessive Alport syndrome in English cocker spaniel dogs Kidney International 54 706-719

Lefebvre $\mathbf{O}$, Sorokin L, Kedinger $\mathbf{M}$ and Simon-Assmann $\mathbf{P}$ (1999) Developmental expression and cellular origin of the laminin alpha2, alpha4, and alpha5 chains in the intestine Developmental Biology $\mathbf{2 1 0}$ 135-150

McArthur ME, Irving-Rodgers HF, Byers S and Rodgers RJ (2000) Identification and immunolocalisation of decorin, versican, perlecan, nidogen, and chondroitin sulfate proteoglycans in bovine small ovarian follicles Biology of Reproduction 63 913-924

Martinez-Hernandez A and Amenta PS (1983) The basement membrane in pathology Laboratory Investigation 48 656-677

Oliviero P, Chassagne C, Salichon N, Corbier A, Hamon G, Marotte F, Charlemagne D, Rappaport L and Samuel JL (2000) Expression of laminin alpha2 chain during normal and pathological growth of myocardium in rat and human Cardiovascular Research 46 346-355

Rodgers HF, Irvine CM, van Wezel IL, Lavranos TC, Luck ML, Sado Y, Ninomiya $Y$ and Rodgers RJ (1998) Distribution of the $\alpha 1$ to $\alpha 6$ chains of type IV collagen in bovine follicles Biology of Reproduction 59 1334-1341

Rodgers RJ, Lavranos TC, van Wezel IL and Irving-Rodgers HF (1999) Development of the ovarian follicular epithelium Molecular and Cellular Endocrinology 151 171-179

Rodgers RJ, Irving-Rodgers HF and van Wezel IL (2000) Extracellular matrix in ovarian follicles Molecular and Cellular Endocrinology 163 73-79

Rodgers RJ, Irving-Rodgers HF, van Wezel IL, Krupa M and Lavranos TC (2001) Dynamics of the membrana granulosa during expansion of the ovarian follicular antrum Molecular and Cellular Endocrinology 171 41-48

Sado Y, Kagawa M, Kishiro Y, Sugihara K, Naito I, Seyer JM, Sugimoto M, Oohashi T and Ninomiya Y (1995) Establishment by the rat lymph node method of epitope-defined monoclonal antibodies recognising the six different $\alpha$ chains of human type IV collagen Histochemistry and Cell Biology 104 267-275

Sanes JR and Chiu AY (1983) The basal lamina of the neuromuscular junction Cold Spring Harbor Symposia for Quantitative Biology 48 667-678

Singh J and Adams GP (2000) Histomorphometry of dominant and subordinate bovine ovarian follicles Anatomical Record 258 58-70

Sorokin LM, Pausch F, Durbeej M and Ekblom P (1997) Differential expression of five laminin alpha (1-5) chains in developing and adult mouse kidney Developmental Dynamics $210446-462$

Vajsar J, Ackerley C, Chitayat D and Becker LE (2000) Basal lamina abnormality in the skeletal muscle of Walker-Warburg syndrome Pediatric Neurology 22 139-143

van Wezel IL and Rodgers RJ (1996) Morphological characterization of bovine primordial follicles and their environment in vivo. Biology of Reproduction 55 1003-1011

van Wezel IL, Rodgers HF and Rodgers RJ (1998) Differential localization of laminin chains in the bovine follicle Journal of Reproduction and Fertility 112 267-278

van Wezel IL, Krupa M and Rodgers RJ (1999) Development of the membrana granulosa of bovine antral follicles: structure, location of mitosis and pyknosis, and immunolocalization of involucrin and vimentin Reproduction, Fertility and Development 11 37-48

Vracko R (1974) Basal lamina layering in diabetes mellitus. Evidence for accelerated rate of cell death and cell regeneration Diabetes 23 94-104

Received 29 June 2001.

First decision 21 August 2001.

Accepted 2 October 2001. 